\title{
Understanding the selective area growth of GaN nanocolumns by MBE using Ti nanomasks
}

\author{
A. Bengoechea-Encabo ${ }^{a}, *$, F. Barbagini ${ }^{a}, S$. Fernandez-Garrido ${ }^{a}$, J. Grandal $^{a}$, J. Ristic $^{\text {a }}$, \\ M.A. Sanchez-Garcia ${ }^{a}$, E. Calleja ${ }^{a}$, U. Jahn ${ }^{b}$, E. Luna ${ }^{b}$, A. Trampert ${ }^{b}$ \\ a ISOM and Dpt. Ing. Electronica, ETSIT, Universidad Politécnica, 28040 Madrid, Spain. \\ ${ }^{\mathrm{b}}$ Paul-Drude-Institut für Festköperelektronik, Hausvogteiplatz 5-7, 10117 Berlin, Germany
}

A R T I C L E I N F O

Keywords:

A3. MBE

A3. Selective area growth

B1. GaN nanocolumns

\begin{abstract}
A B S T R A C T
The influence of the substrate temperature, III/V flux ratio, and mask geometry on the selective area growth of GaN nanocolumns is investigated. For a given set of growth conditions, the mask design (diameter and pitch of the nanoholes) is found to be crucial to achieve selective growth within the nanoholes. The local III/V flux ratio within these nanoholes is a key factor that can be tuned, either by modifying the growth conditions or the mask geometry. On the other hand, some specific growth conditions may lead to selective growth but not be suitable for subsequent vertical growth. With optimized conditions, ordered GaN nanocolumns can be grown with a wide variety of diameters. In this work, ordered GaN nanocolumns with diameter as small as $50 \mathrm{~nm}$ are shown.
\end{abstract}

\section{Introduction}

The epitaxial growth of III-nitride nanocolumns (NCs) has attracted much interest during the last years $[1,2]$. NCs are strainand dislocation-free [3], thus being ideal candidates to study basic materials properties at nanometric scale, and also have a strong potential for a wide variety of applications. Self-assembled IIInitride NCs can be grown on different substrates $[1,2,4]$ and their controlled coalescence is used to produce strain-free pseudosubstrates with reduced defect densities [5]. III-nitride nanocolumnar complex hetero-structures have been grown by molecular beam epitaxy (MBE), like LEDs [6-8] and nanocavities [9], which, due to the absence of extended defects, are very efficient.

Unfortunately, self-assembled growth is a random process with a rough control of the NC density and diameter [10,11], which is a drawback for a direct application of NCs to real devices. Indeed, fluctuations of density and diameter would lead to strong electrical and optical properties dispersion. An ensemble of nanodevices connected in parallel with such properties dispersion may lead to low emission efficiency due to localized current injection. An inhomogeneous distribution of NC heights would make the device processing difficult or inefficient. When InGaN quantum disks (QDiscs) are embedded in GaN NCs diameter fluctuations may lead to variations, both of QDisc thickness and In

\footnotetext{
* Corresponding author.

E-mail address: abengo@rut.die.upm.es (A. Bengoechea-Encabo).
}

composition, which would strongly affect the optical properties. Selective area growth (SAG) [12-14] represents an efficient way to overcome such problems, in which the substrate is masked with an array of nanoholes.

Growth conditions must be carefully tuned to achieve selectivity, meaning growth only within the holes while avoiding it on the mask (usually $\mathrm{Ti}, \mathrm{Mo}, \mathrm{SiN}$, and $\mathrm{SiO}_{2}$ ). Selectivity depends critically on the sticking coefficients, diffusion lengths, and desorption rates of the metal ad-atoms, as well as on the geometry of the nanohole array in the mask. This growth process differs considerably from the self-assembled one, where the main driving force for the spontaneous formation of NC is the lattice mismatch between the substrate and the NCs material [10,11].

This work studies the effects of the MBE growth parameters (substrate temperature and III/V flux ratio) and nanohole mask geometry on the selective area growth on GaN templates covered with a Ti nanohole mask. The main objective is to understand the growth process and to establish optimal conditions to achieve and to control selectivity and homogeinity. Besides the SAG of GaN NC, we report on a growth regime leading to pyramidal nanostructures inside the nanoholes and characterized by the inhibition of the vertical growth, which has not been reported in previous works on GaN SAG by MBE [12-14]. The present paper also remarks the key parameters to produce the transition from this growth regime to a vertical growth one. It is also important to point out that, in the present case, precise III/V flux ratios are specified, unlike in previous works. All these aspects together contribute to a deeper understanding of the GaN SAG by MBE. 


\section{Experimental procedure}

Nanohole masks were fabricated by e-beam lithography (EBL) on commercial (0001) GaN templates grown by MOVPE on sapphire (Lumilog). A $5 \mathrm{~nm}$ thick Ti layer was deposited by e-beam evaporation. A positive resist ZEP520A was used and the process optimized to obtain arrays of nanoholes with diameters ranging from 50 up to $200 \mathrm{~nm}$ and a pitch (distance between centers of nanoholes) varying from 100 to $300 \mathrm{~nm}$. Due to the critical importance of the diffusion length and sticking coefficient of $\mathrm{Ga}$ ad-atoms on the metallic layer the roughness of the deposited Ti layer was measured by AFM prior to the growth, yielding a value of $0.4 \mathrm{~nm}(\mathrm{rms})$ in an area of $1 \times 1 \mu \mathrm{m}^{2}$. The Ti layer was etched down by reactive ion etching using a $\mathrm{O}_{2} / \mathrm{CF}_{4}$ plasma at $10^{-2}$ mbar. The etching depth was measured by atomic force microscopy (AFM) to be between 7 and $10 \mathrm{~nm}$; thus, the GaN template was also etched in the range of some $2-5 \mathrm{~nm}$. Growths were carried out in a radio frequency (RF) plasmaassisted molecular beam epitaxy (PAMBE) system (Riber Compact 21 ). The substrate temperature was monitored with a thermocouple located at the growth stage. The $\mathrm{Ga}$ and $\mathrm{N}$ fluxes were calibrated in equivalent $(00001)$ GaN growth rate units for compact layers (nm/min). To report molecular fluxes in $(\mathrm{nm} /$ min) units is the standard procedure used in nitrides MBE growth maps $[15,16]$. Since the temperature values for selective growth are quite high $\left(\geq 900^{\circ} \mathrm{C}\right.$ ) nitridation of the Ti mask, prior to the growth, was needed to prevent its degradation. The nitridation process was similar to that proposed by Sekiguchi et al. [12], following two steps: a first one at low temperature $\left(460^{\circ} \mathrm{C}\right.$ for $10 \mathrm{~min})$ and a second one at high temperature $\left(880^{\circ} \mathrm{C}\right.$ for $\left.3 \mathrm{~min}\right)$. The plasma source during nitridation was set to $580 \mathrm{~W}$ at nitrogen flux of $1.2 \mathrm{sccm}$, which corresponds to an equivalent stoichiometric GaN growth rate higher than $30 \mathrm{~nm} / \mathrm{min}$. Electron energy loss spectroscopy measurements (not shown) prove the formation of TiN, more stable than Ti at high temperatures. This nitridation process (avoiding degradation at high temperature) together with the assessment of a low Ti mask surface roughness (below $0.5 \mathrm{~nm}$, rms value, which favors the Ga diffusion) is the key starting point needed to achieve SAG

Scanning electron microscopy (SEM) images were obtained with a CRESTEC CABL-9500C system. Atomic force microscopy (AFM) images were taken with a Nanoscope microscope (MMAFM-2) and processed with Nanotec software WSxM [17]. Transmission electron microscopy (TEM) studies were carried out with JEM 3010 and 2100F microscopes.

\section{Experimental results and discussion}

Selective growth of GaN NCs was studied in a set of samples, using EBL masks with nanoholes diameter around $150 \mathrm{~nm}$ and pitch $300 \mathrm{~nm}$. The duration of the growths is $30 \mathrm{~min}$, growth temperatures vary from 900 to $960^{\circ} \mathrm{C}$, and nominal $111 / \mathrm{V}$ flux ratios from 0.7 to 2.7 . These nominal flux ratios are quite high when compared to typical ones for self-assembled NCs (III/V $\ll 1)$ [10]. However, considering the high temperatures needed for SAG, the actual III/V ratios are much smaller than the nominal ones because of the very strong Ga desorption and GaN decomposition rates. On the other hand, these high temperatures are needed for SAG in order to enhance both Ga surface diffusion (higher probability for Ga ad-atoms to reach the nanoholes) and Ga surface desorption to avoid or minimize the formation of GaN NCs on the mask [12]. This is indeed a very delicate trade-off. Fig. 1 shows the corresponding plan-view SEM images. For a nominal III/V flux ratio of $0.7(\mathrm{Ga}=10 \mathrm{~nm} / \mathrm{min} ; \mathrm{N}=15 \mathrm{~nm} / \mathrm{min})$ and $940{ }^{\circ} \mathrm{C}$ (Fig. 1a) there are GaN NCs both on the Ti mask and

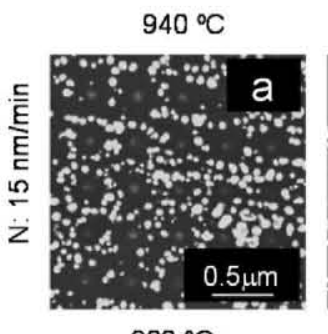

$900^{\circ} \mathrm{C}$

$\mathrm{N}: 5 \mathrm{~nm} / \mathrm{min}$
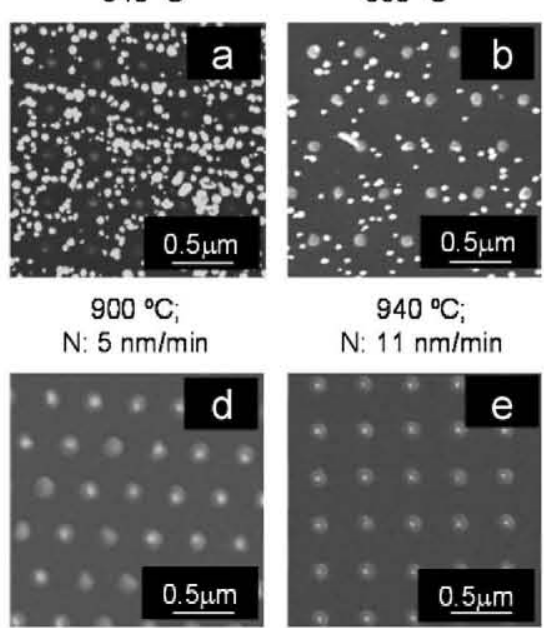

$\mathrm{N}: 11 \mathrm{~nm} / \mathrm{min}$

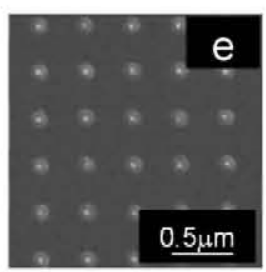

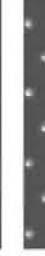

$960^{\circ} \mathrm{C}$

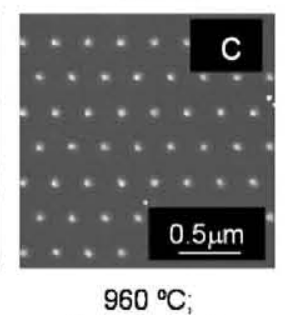

$\mathrm{N}: 15 \mathrm{~nm} / \mathrm{min}$

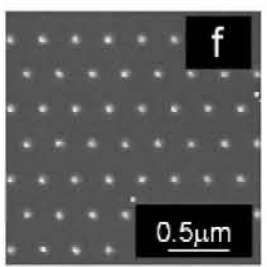

Fig. 1. Plan-view SEM images of a series of samples grown for $30 \mathrm{~min}$ on EBL Ti masks with nanoholes of around $150 \mathrm{~nm}$ diameter and $300 \mathrm{~nm}$ pitch. Growth temperature and nominal III/V ratio are varied (different $\mathrm{N}$ fluxes for a given $\mathrm{Ga}$ flux) in order to check their effect on the NC nucleation selectivity.

within the nanoholes (no selectivity). A substrate temperature increment of $10^{\circ} \mathrm{C}$ is not enough to achieve full selectivity (Fig. 1b). Finally, at $960^{\circ} \mathrm{C}$ perfect selectivity was achieved (Fig. 1c). At even higher temperature, growth is no longer possible under these conditions because of the very strong Ga desorption and GaN decomposition rates.

From the point of view of selectivity the growth temperature and nitrogen flux (or reciprocal III/V ratio) act in opposite ways [10], since a higher growth temperature promotes the diffusion and desorption of Ga ad-atoms (better selectivity) while a higher nitrogen flux increases the probability of GaN formation on the mask before Ga desorption occurs (worse selectivity) [18]. Thus the growth conditions to obtain the same results are not unique, being achievable at lower substrate temperature by simply increasing the III/V ratio. This is shown in Fig. 1d, e, and f, which correspond to III/V nominal ratios of $3.2(\mathrm{Ga}=16 \mathrm{~nm} / \mathrm{min}$; $\mathrm{N}=5 \mathrm{~nm} / \mathrm{min}) ; 0.9(\mathrm{Ga}=10 \mathrm{~nm} / \mathrm{min} ; \mathrm{N}=11 \mathrm{~nm} / \mathrm{min}) ;$ and 0.7 $(\mathrm{Ga}=10 \mathrm{~nm} / \mathrm{min} ; \mathrm{N}=15 \mathrm{~nm} / \mathrm{min})$, respectively.

A second series of samples was grown at $960{ }^{\circ} \mathrm{C}$ with a nominal III/V flux ratio of $1.23(\mathrm{Ga}=16 \mathrm{~nm} / \mathrm{min} ; \mathrm{N}=13 \mathrm{~nm} / \mathrm{min})$ for $30 \mathrm{~min}$. The nanohole diameter varied from 200 to $50 \mathrm{~nm}$. For wide nanoholes $(200 \mathrm{~nm})$, GaN pyramids are formed selectively (Fig. 2a) and facets at $43^{\circ}$ from the $c$-plane that correspond to $\left\{\begin{array}{llll}1 & -1 & 0 & 2\end{array}\right\}$ planes ( $r$-planes) as measured by TEM and AFM (Fig. 3). Surprisingly, when the growth time is increased to 90 min under the same growth conditions and mask geometry as for the sample in Fig. 2a, similar GaN pyramidal nanostructures are observed but no further vertical growth occurs (i.e. same pyramid height for 30 or $90 \mathrm{~min}$ ) as if the evolution to complete and vertical NCs was inhibited. Consonni et al. [19] reported recently on the shape transition from semi-spherical islands to full NCs through intermediate pyramids (first truncated, then, full ones) in self-assembled GaN NCs grown on AlN buffered Si. Minimization of the total free energy (strain and surface) per unit volume predicts a specific shape, the transition being from pyramids to full NCs driven by dislocations generation (release of elastic strain energy). However, the fact that, in our case there is no strain involved in the homoepitaxial growth (no dislocation generation as observed by TEM measurements) might be a reason to inhibit the shape evolution from pyramidal islands to full NCs. It is also known that for homoepitaxial growth of GaN the layer morphology is governed by the growth regime, either "Ga-stable" 
or "N-stable" [20,21]; when occurring under "N-stable" conditions the surface morphology is faceted. In this case, the inhibition of the vertical growth previously mentioned for GaN NCs could be related with a higher decomposition rate of these facets compared to the growth rate on them under the specific conditions used (growth barrier).

When reducing the nanohole diameter to $50 \mathrm{~nm}$ (pitch $100 \mathrm{~nm}$ ), while keeping the growth conditions and time as those for samples in Fig. 2a, the main difference is that instead of pyramidal islands, well developed NCs are obtained, having a height of $200 \mathrm{~nm}$ (Fig. 2b). This drastic change in morphology, which apparently depends only on the nanohole diameter (from 200 to $50 \mathrm{~nm}$ ), may be understood considering that, for the smaller nanohole, the density of Ga ad-atoms within it (including Ga atoms arriving by diffusion) is much higher. This means a
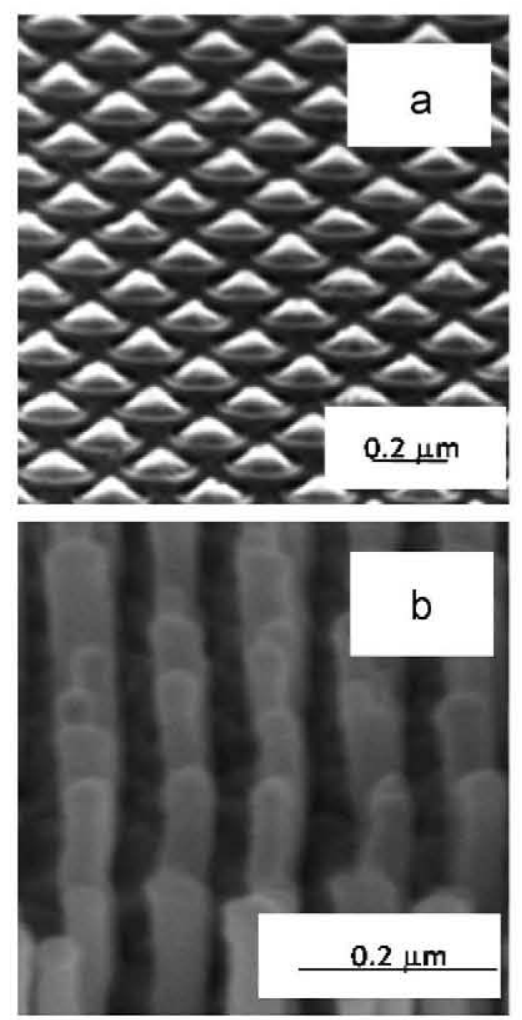

Fig. 2. Bird-eye SEM images of samples at the growth conditions of Fig. 1c, changing nanohole diameter; $200 \mathrm{~nm}$ (a) and $50 \mathrm{~nm}$ (b). higher (local) III/V ratio within the nanohole, but not on the mask, so that selectivity is preserved. This higher III/V ratio within the nanohole may modify kinetics leading to a NC stable shape with flat tops (c-plane) as shown in Fig. $2 \mathrm{~b}$ (poor definition and blurry aspect are due to charge effects). The contribution of diffuse Ga, from the surrounding of a nanohole, to the local III/V ratio within the nanohole is related to the Ga re-evaporation and diffusion length on top of a nitrided Ti surface. These values are difficult to determine accurately in our current MBE system but a simple geometrical consideration can be taken to compare the different contributions of $\mathrm{Ga}$ to the nanohole, obtained by modifying the diameter of the nanohole. If we consider that the Ga diffusion value is $l$ (for a given substrate, temperature, and nitrogen flux) and the diameter of the nanohole is $d$, following simple geometrical calculations, the amount of Ga per unit area that diffuses into the nanohole is proportional to $l(l+d) /\left(d^{2}\right)$ (diffusion area/ nanohole area). Although $l$ is difficult to estimate, if we assume a low diffusion length value, such as $20 \mathrm{~nm}$, the diffuse Ga contributing to the local III/V ratio within the nanohole is 5 times larger in the case of holes of $50 \mathrm{~nm}$ of diameter as compared with the $200 \mathrm{~nm}$ holes. This difference is even larger as the value of the diffusion length increases.

A clarifying experiment followed, in which a $150 \mathrm{~nm}$ nanohole diameter EBL Ti mask was used keeping the same growth conditions as those used for the sample in Fig. $1 \mathrm{~d}(\mathrm{Ga}=16 \mathrm{~nm} / \mathrm{min}$ and $\mathrm{N}=5 \mathrm{~nm} / \mathrm{min}$ ). In this case the growth temperature was $900{ }^{\circ} \mathrm{C}$, a decrease of $60^{\circ} \mathrm{C}$ with respect to the previous experiment with reduced nanoholes diameter, and the growth time was $1 \mathrm{~h}$. Results are most relevant since well developed GaN NCs are obtained (Fig. 4a), although semi-polar facets are still present on top. TEM results illustrate the high quality of the GaN NCs, without extended
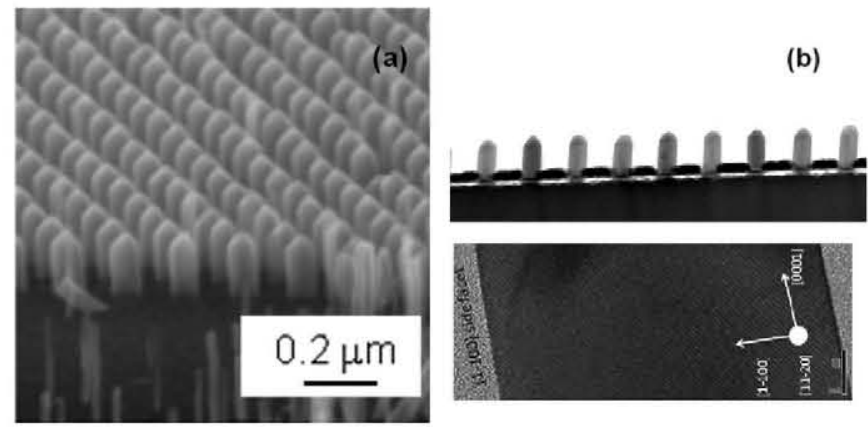

Fig. 4. Bird-eye SEM (a) and TEM (b) images of ordered NCS on an array of $150 \mathrm{~nm}$ diameter nanoholes and $275 \mathrm{~nm}$ pitch. Growth conditions are the same as those in Fig. 1d (b).
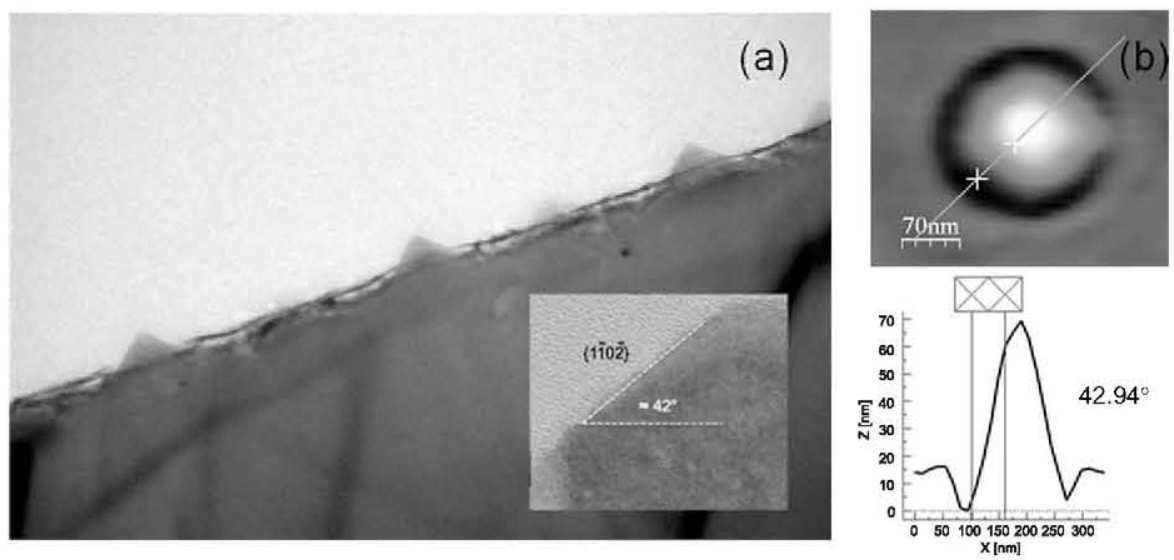

Fig. 3. TEM (a) and AFM (b) of sample in Fig. 2a, with nanohole diameter $200 \mathrm{~nm}$. Semi-polar $\{1-102\}$ planes are predominant facets of the pyramids. 
defects and with well defined lateral $m$-planes (Fig. $4 \mathrm{~b}$ ). Due to the low N flux no lateral growth is observed, so once the NCs are formed within the nanoholes, they grow exclusively in the vertical direction. Moreover, in the case of the thin NCs of Fig. 2b, even under a $N$ flux of $13 \mathrm{~nm} / \mathrm{min}$, no lateral growth is detected, probably due to the very high growth temperatures. So in both cases, NCs are grown following the mask dimensions. These results allow building up of a consistent picture to explain the SAG process. Aside from selectivity achievement, the local III/V ratio within the nanoholes plays a crucial role. If the local III/V ratio within nanoholes is too low, NCs are formed producing pyramidal facets ( $r$-planes) that show a reduced growth rate so that full pyramids develop (no $c$-plane on top) and the vertical growth is inhibited (Fig. 2a). If the nanohole diameter is strongly reduced while keeping the same growth conditions the local III/V ratio within nanoholes is much higher, yielding full NCs with vertical sidewalls ( $m$-planes; Fig. $2 \mathrm{~b}$ ). The same results can be achieved using wider nanoholes $(150 \mathrm{~nm})$ but reducing the temperature (to $900{ }^{\circ} \mathrm{C}$ ), which means a much higher local III/V ratio within the nanoholes (lower Ga desorption), as shown in Fig. 4. However, in this case, NCs do not have flat tops. This may be also understood considering that in this case the local III/V ratio within the nanoholes is not high enough to avoid the presence of facets ( $r$-planes) but high enough to enhance the growth rate on them, so that NCs grow vertically but keeping the facets. The control of the top surface of the SAG GaN NCs is a relevant issue, moreover since it will influence drastically the optical and electrical properties of any active region (i.e. quantum disk, for example) that is embedded in the GaN NC. The analysis of the morphology of the top surface of the $\mathrm{NC}$ and its control is an issue under current study and it will be the topic of an upcoming publication.

\section{Summary}

As a summary, for homoepitaxial SAG of GaN NCs two main factors should be considered: (i) facet generation upon initial formation of the $\mathrm{NC}$ and (ii) the inherent difficulty (barrier) to grow on facets ( $r$-planes). As mentioned before a given local III/V ratio within nanoholes may be high enough to prevent (i) and then obviously (ii), or not enough to prevent (i) but allowing to overcome the growth barrier due to (ii). As for the last point, the experimentally observed difficulty to grow on GaN $r$-planes is not new, and it has been observed in many cases referring to different epitaxial growth techniques. In some cases the tendency of $\mathrm{N}$-polar $r$-planes to be passivated by hydrogen has been invoked [22]. In other cases a lower reactivity of these surfaces (bonding, flatness, etc.) has been suggested, but no definite explanation has been given so far, thus remaining an open question.

In conclusion the growth conditions needed to achieve SAG of GaN NCs by PAMBE on Ti masked GaN templates were studied. Aside from the fact that Ti masks must be nitridated to withstand the high growth temperatures, SAG is achieved under different conditions where the $\mathrm{Ga}$ ad-atom surface diffusion length and desorption rates are adequate to the specific mask geometry. Growth temperature and III/V ratio play opposite roles to achieve selectivity. Once selectivity is achieved at the stage of initial formation of the NCs the subsequent shape evolution depends strongly on the local III/V ratio, which can be adjusted by the growth temperature and mask design.

\section{Acknowledgment}

We acknowledge partial funding by National Projects CAM P2009/ESP-1503, MICINN-PLE2009-0023, MICINN-MAT-200804815, MICINN-Consolider CSD2006-19, and by UE CP-IP 228999-2 (SMASH).

\section{References}

[1] M. Yoshizawa, A. Kikuchi, M. Mori, N. Fujita, K. Kishino, Jpn. J. Appl. Phys. 36 (1997) L459.

[2] M.A. Sanchez-García, E. Calleja, E. Monroy, F.J. Sanchez, F. Calle, E. Muñoz R. Beresford, J. Cryst. Growth 183 (1998) 23

[3] E. Calleja, J. Ristić, S. Fernández-Garrido, L. Cerutti, M.A. Sánchez-García, J. Grandal, A. Trampert, U. Jahn, G. Sánchez, A. Griol, B. Sánchez, Phys. Status Solidi (b) 244 (8) (2007) 2816.

[4] L. Cerutti, J. Ristic, S. Fernández-Garrido, E. Calleja, A. Trampert, K.H. Ploog, S. Lazic, J.M. Calleja, Appl. Phys. Lett. 88 (2006) 213114

[5] Z. Bougrioua, P. Gibart, E. Calleja, U. Jahn, A. Trampert, J. Ristic, M. Utrera, G. Nataf, J. Cryst. Growth 309 (2007) 113.

[6] H. Sekiguchi, K. Kato, J. Tanaka, A. Kikuchi, K. Kishino, Phys. Status Solidi (a) 205 (2008) 1067.

[7] Hon-Way Lin, Yu-Jung Lu, Hung-Ying Chen, Hong-Mao Lee, Shangjr Gwo, Appl. Phys. Lett. 97 (2010) 073101.

[8] W. Guo, M. Zhang, A. Banerjee, P. Bhattacharya, Nano Lett. 10 (2010) 3355.

[9] J. Ristić, E. Calleja, A. Trampert, S. Fernández-Garrido, C. Rivera, U. Jahn, K.H. Ploog, Phys. Rev. Lett. 94 (2005) 146102.

[10] S. Fernández-Garrido, J. Grandal, E. Calleja, M.A. Sánchez-García, D. LópezRomero, J. Appl. Phys. 106 (2009) 126102.

[11] J. Ristic, E. Calleja, S. Fernandez-Garrido, L. Cerutti, A. Trampert, U. Jahn, K.H. Ploog, J. Cryst. Growth 310 (18) (2008) 4035

[12] H. Sekiguchi, K. Kishino, A. Kikuchi, Appl. Phys. Express 1 (2008) 124002.

[13] K. Kishino, H. Sekiguchi, A. Kikuchi, J. Cryst. Growth 311 (2009) 2063.

[14] H. Sekiguchi, K. Kishino, A. Kikuchi, Appl. Phys. Lett. 96 (2010) 231104.

[15] B. Heying, R. Averbeck, L.F. Chen, E. Haus, H. Riechert, J.S. Speck, J. Appl. Phys 88 (2000) 1855

[16] G. Koblnüller, S. Fernández-Garrido, E. Calleja, J.S. Speck, Appl. Phys. Lett. 91 (2007) 161904

17] I. Horcas, R. Fernandez, J.M. Gomez-Rodriguez, J. Colchero, J. Gomez-Herrero, A.M. Baro, Rev. Sci. Instrum. 78 (2007) 013705 Software Nanotec at.

[18] S. Fernández-Garrido, G. Koblmüller, E. Calleja, J.S. Speck, J. Appl. Phys. 104 (2008) 033541 .

[19] V. Consonni, M. Knelangen, L. Geelhaar, A. Trampert, H. Riechert, Phys. Rev. B $81(2010) 085310$.

[20] E.J. Tarsa, B. Heying, X.H. Hu, P. Fini, S.P. DenBaars, J.S. Speck, J. Appl. Phys. 82 (1997) 5472.

[21] C. Adelmann, J. Brault, D. Jalabert, P. Gentile, H. Mariette, G. Mula, B. Daudin J. Appl. Phys. 91 (2002) 9638.

22] S.F. Li, S. Fuendling, X. Wang, S. Merzsch, M.A.M. Al- Suleiman, J.D. Wei, H.-H. Wehmann, A. Waag, W. Bergbauer, M. Strassburg, Polarity and its influence on growth mechanism during MOVPE growth of GaN nanorods, unpublished. 